\title{
Turbulent mixing in a non-magnetic corona: Physical and numerical factors
}

\begin{abstract}
R. Grappin and J. Léorat
DAEC, Observatoire de Paris, Université Paris VII, CNRS (UMR 8631), 92195 Meudon Cedex, France and ASCI, France

Received 8 June 2000 / Accepted 5 October 2000

Abstract. We study the shear instability and turbulent mixing of radial streams originating in thermal inhomogeneities of a non-magnetic corona. This is achieved by solving the time-dependent axisymmetric Navier-Stokes equations within a central gravitational field and polytropic index unity. Cold regions lead to cold wakes, which can become unstable and eventually lead to turbulent mixing (Grappin et al. 1997). The aim of this work is to understand the conditions for the development of the instability, which in many aspects is close to the standard Kelvin-Helmholtz instability, but in which several features specific to the solar wind intervene. A prerequisite is to reach a sufficiently high Reynolds number, i.e., to reduce damping (compared to molecular viscous damping), without generating spurious noise (mainly the Gibbs effect) which could artificially trigger the instability. This is achieved via a nonlinear filtering scheme, first developed here on the simpler problem of shock formation (Burgers equation), and then applied to shear flows. We conclude that cold wakes generated by cold regions in the corona are made unstable by the pinching effect of surrounding hotter streams. The pinching effect, and hence the instability itself, is strongly dependent on the mean temperature.
\end{abstract}

Key words. hydrodynamics - instabilities - turbulence - methods: numerical - Sun: corona - (Sun:) solar wind

\section{Introduction}

The solar wind observed in situ in the ecliptic plane is a mixture of fast and slow streams which could feed, via the Kelvin-Helmholtz instability, the observed well-developed spectrum (Coleman 1968). The very origin of the slow wind has been ascribed by Habbal et al. (1997) to reside in the streamer stalks close to the sun. Einaudi et al. (1999) have recently given consistency to this hypothesis by modeling numerically the instability of homogeneous shear flows in the vicinity of a current sheet. The possibility that turbulence in the ecliptic plane could be comparable to a wind tunnel turbulence, with the magnetic streamer belt playing the role of a magnetic grid at the inlet of the tunnel was suggested by Mangeney et al. (1991).

There is, in fact, another (non-magnetic) grid which may provide the seed of the micro-stream structure, and possibly is at the origin of the turbulent spectrum, namely the thermal structure of the corona. Indeed, as has been shown in Grappin et al. (1997), cold regions are at the origin of cold, slow streams which, being pinched by hot streams, are eventually destabilized. The destabilization of stream shear was thus proved to be possible in the vicinity of the acceleration region, while on the other hand, in

Send offprint requests to: R. Grappin,

e-mail: Roland.Grappin@obspm.fr the developed supersonic wind, the spherical expansion severely hampers the growth of such instabilities (Grappin et al. 1993; Grappin \& Velli 1996; Grappin 1996).

The main aim of this work is not to make quantitative predictions of the distance of turbulent mixing in the solar wind, but to show the specific properties of shear flows in the accelerating region of the solar wind, in the absence of a magnetic field. This may be considered as an approach to the problem of turbulent non-magnetic winds, but we prefer to view it as a first step towards a study of the turbulent magnetohydrodynamic (MHD) wind.

What are the physical factors controlling the instability of shear flows? In the homogeneous version of the problem, the initial shear flow amplitude can be controlled; both its amplitude and the transverse velocity determine the onset and spatial development of the instability. The solar wind version of the problem is different, because the amplitude of the shear cannot be directly controlled, since, as the Parker transonic solution is an attractor, the radial velocity profile is determined by the local temperature (Grappin et al. 1996; Velli 1994). Hence, in the present problem, the two parameters are: the seed transverse velocity, and the temperature jump at the inner boundary, which (dynamically) leads to the stream shear. The role of these two parameters will be studied. 
Another physical parameter is clearly playing a role, namely the Reynolds number, which measures the ratio of inertial forces over dissipative terms. If it is too low, the instability onset is very slow, and in the case of the solar wind, the flow becomes stabilized by the expansion. Numerical practice shows that it is very difficult to destabilize shear flows in the accelerating region by using classical molecular dissipation terms, be it only because of the increase of the characteristic mesh size with radial distance: all shear is dissipated in the domain close to the bottom boundary close to the sun. In principle, it is possible to increase the Reynolds number by increasing the resolution, but in practice, it is much more efficient to use numerical filtering. This was used for instance in Grappin et al. (1997). However, numerical filtering generally introduces artificial Gibbs-like oscillations. These numerical oscillations have several drawbacks: a) they prevent measuring correctly the jump amplitude (in velocity or temperature) and thus the onset of turbulent mixing; b) when the discontinuity is tangential (wakes, shear flows) and the stability of the wake depends critically on the amplitude of the gradients, one may suspect that the Gibbs oscillations trigger the instability, and hence prevent the correct measurement of the threshold conditions. We will see several examples of this in the following sections.

The problem of minimizing the dissipation at a fixed resolution without introducing artificial oscillations may be solved by using ENO schemes (see Harten et al. 1997). Also, nonlinear viscosities preserving the thermodynamic laws of the dissipation function have been proposed for example by Passot \& Pouquet (1988) for compressible flows, and Siregar et al. (1995) for MHD flows. As we shall see, such nonlinear (or hyper-) viscosities are generally more demanding in computer time than is molecular viscosity.

We shall show in this paper that one can define a composite (non-linear) filter allowing a strong decrease of the dissipation, without any additional computing time compared to standard molecular dissipation, and with a zero or low level of artificial oscillations. The filtering scheme will be built up first considering Burgers equation, and then applied with some changes to the stream instability problem.

The plan is the following: Section 2 is devoted to presenting the numerical method for the spatial and temporal schemes, the boundary conditions, and introducing the nonlinear filter developed in the other sections. Section 3 develops the nonlinear filter in the case of the Burgers equation (shock problem), and Sect. 4 reports the results of numerical simulations of the turbulent mixing problem in the shear flows induced by temperature inhomogeneities in a non-magnetic solar corona. Section 5 contains our discussion.

\section{Method}

\subsection{The Gibbs effect revisited}

To illustrate the problem and introduce the method, let us consider the Burgers equation, which describes the formation of a shock starting from a regular solution (say a sine). The solution can be computed analytically, and has been extensively used as a test for several numerical algorithms (Basdevant et al. 1986). The equation reads:

$\partial u / \partial t+u \partial u / \partial x=\nu \partial^{2} u / \partial x^{2}$.

Starting from $u=U^{0} \sin x$, the profile will steepen, i.e. the velocity jump $U^{0}$ will occur on a distance interval which decreases with time. This will increase the amplitude of the dissipation term (rhs of Eq. (1)), up to a value where it balances the non-linear advection term. At a given resolution, a minimal viscosity is needed for the shock to be well defined (i.e. to have a width larger than the mesh size). One can define a critical viscosity $\nu^{0}$ by writing that the dissipative time at the grid scale equals the advective time: $\nu k_{\max }^{2} \simeq k_{\max } U^{0}$, where $k_{\max }=\pi / h$ ( $h$ being the mesh size) is the maximum wave number:

$\nu^{0}=U / k_{\max }$.

With this value of the viscosity, the width of the quasistationary shock should be of the order of a mesh size $h$. One finds that the minimum viscosity for the shock to be resolved correctly is in practice somewhat larger than the critical value $\nu^{0}: \nu=1.5 \nu^{0}$. The numerical evolution shows two phases (Fig. 1, top). The pre-shock phase shows a substantial dissipation, which can be reduced by increasing the resolution. On the contrary, when the resolution is high enough, the dissipation during the second phase is independent of the resolution, when we vary the resolution and keep the viscosity minimal value. Note that if we assign a too low value to the viscosity (below $1.5 \nu^{0}$ ), the shock width decreases too much, parasitic sawtooth oscillations appear around the shock with a wavelength comparable to the grid size, and then rapidly dominate the whole solution.

The diffusive term in the Burgers equation is actually equivalent to a filter by which we would multiply the Fourier transform of the ideal (non diffusive) solution at each time step; this filter reads:

$\exp \left(-\nu k^{2} \mathrm{~d} t\right) \simeq \exp \left[\left(-\left(k / k_{\max }\right)^{2}\right]\right.$.

The rhs of Eq. (3) is obtained by choosing (as required for stability) a time step $\mathrm{d} t$ computed on the diffusive time at grid scale, namely $\mathrm{d} t=1 /\left(\nu k_{\max }^{2}\right)$. This is valid if $\nu \geq \nu_{0}$; otherwise, the time step must be computed on the advective time, and the filtering at a given wave number $k$ is weaker: $\exp \left[\left(-\nu / \nu_{0}\right)\left(k / k_{\max }\right)^{2}\right]$. To obtain a reduced dissipation during the shock building phase, we must replace the expression (3) by another one, which dissipates less the small wave numbers. A classical example is provided by the bi-Laplacian $-\nu_{4} \partial^{4} u / \partial x^{4}$ (Basdevant et al. 1986), for which the equivalent filter is:

$\exp \left(-\nu_{4} k^{4} \mathrm{~d} t\right)=\exp \left[\left(-\left(k / k_{\max }\right)^{4}\right]\right.$.

Figure 1 allows to compare the respective solutions of the Burgers equations with a Laplacian (Fig. 1, top) and a bi-Laplacian expression (bottom). In the Laplacian case, 


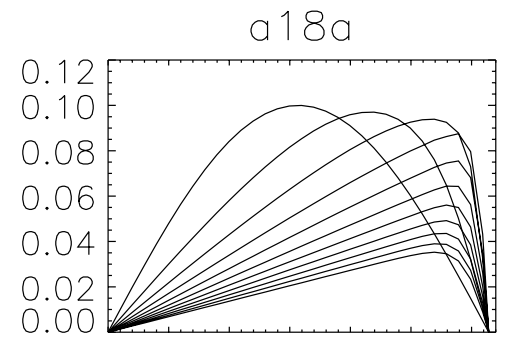

0.00 .51 .01 .52 .02 .53 .0

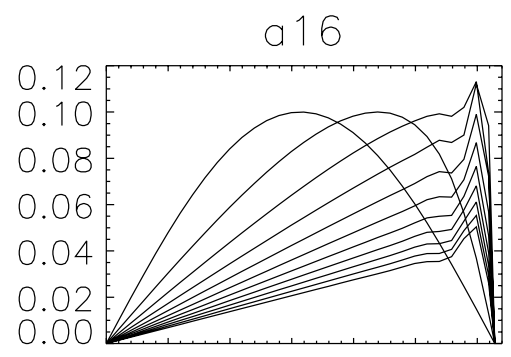

0.00 .51 .01 .52 .02 .53 .0

Fig. 1. Solution of the Burgers equation: evolution of a sinusoidal profile with standard damping (Laplacian, top) and hyperviscosity (bi-Laplacian, bottom)

one sees that the profile is strongly damped during the shock formation phase, since the low resolution, of 64 grid points, imposes a high viscosity. With the bi-Laplacian, one finds empirically that the caution factor is higher than for the Laplacian: the minimum value is $\nu_{4}=4 \nu_{4}^{0}$, where $\nu_{4}^{0}$ corresponds to a diffusive time at grid scale equal to the advective time (cf. Eq. (2)): $\nu_{4}^{0}=U / k_{\max }^{3}$. But with $\nu_{4}=4 \nu_{4}^{0}$, we are forced to adopt a time step four times smaller than the advection time, at least when the temporal scheme is explicit. Moreover, we see in Fig. 1 (bottom) that, although the damping is indeed small during the shock formation phase, the shock once formed is badly described using a bi-Laplacian, since it shows a Gibbslike oscillation which amounts to about $10 \%$ of the total variation.

The preceding filters (Eqs. (3) and (4)) take the form of a dissipative term (Laplacian in Eq. (1), or bi-Laplacian) in the equation of the movement. One can also use, instead of explicit damping terms, direct filters, i.e. filters which are applied at each time step. The interest of direct filters is that they should be stable, i.e., they don't require a reduced time step compared to the advective time, as is the case for the bi-Laplacian. An example of a direct filter is provided by Lele (1992), who uses a compact (implicit), finite differences scheme. If one uses such a filter when integrating the Burgers equation, the result is not better than the one obtained using hyperviscosity, i.e. dissipation is reduced, but the Gibbs effect is very high. Figure 2 shows in Fourier space the Laplacian and bi-Laplacian filters, as well as one of the compact filters of Lele (called "strong" filter in the following, see Eqs. (7) and (9) below). In the top of Fig. 2, the Laplacian and bi-Laplacian filters have their diffusive parameters set to their critical values $\nu^{0}$ and $\nu_{4}^{0}$, obtained by balancing the
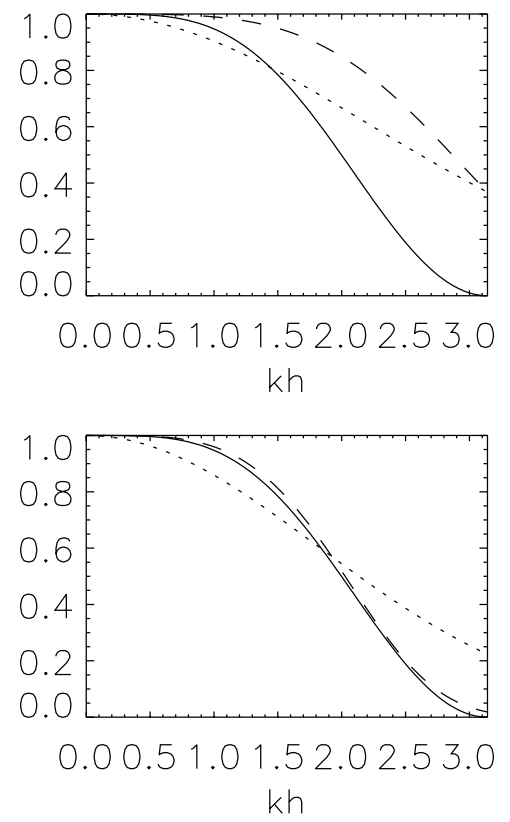

Fig. 2. Three filters versus wave number $k$ ( $h$ is the mesh size). Continuous line: compact filter (Eq. (9)); dotted line: Laplacian; dashed line: bi-Laplacian. Top: diffusive parameters are $\nu=\nu^{0}$ and $\nu_{4}=\nu_{4}^{0}$. Bottom: diffusive parameters are $\nu=1.5 \nu^{0}$ and $\nu_{4}=4 \nu_{4}^{0}$. Note that the bi-Laplacian and the filter are almost superposed in the latter case

diffusive and advective times at the grid scale; in other words, the curves plotted are given by Eqs. (3) and (4). In practice, however, as we already mentioned, a correct grid-scale damping needs diffusion coefficients with values larger than the critical values, namely $\nu_{4}=4 \nu_{4}^{0}$ and $\nu=1.5 \nu^{0}$. The new filtering functions obtained in this way are plotted in Fig. 2 (bottom). One now sees that the bi-Laplacian and the compact filter appear to be almost identical.

Another classical method which allows for substantial reduction of the dissipation in the pre-shock phase consists of using a variable viscosity concentrated in the regions of strong gradients. The simplest solution amounts to a viscosity proportional to the gradient: $\nu=\nu_{1}|\partial u / \partial x|$ (see Roache 1972; Passot \& Pouquet 1988; Siregar et al. 1995). Note that Guermond (2000) uses a slightly different form $\nu=\nu_{\mathrm{G}}|\partial(\hat{u}-u) / \partial x|$, where $\hat{u}$ is the filtered signal. The critical value of $\nu_{1}$ is obtained by normalizing to the maximum of the gradient, $k_{\max } U$ :

$\nu_{1}^{0}=1 / k_{\max }^{2}$.

Figure 3 shows the profile evolution obtained with this method. Note that, in order that the computation proceeds to the end, we had to use a value 5 times larger than the critical value defined above, namely $\nu_{1}=5 \nu_{1}^{0}$. The Gibbs effect is absent, but the figure shows everywhere a numerical noise at the grid scale. If the viscosity is raised higher still, the noise decreases but the Gibbs effect reappears! This solution is thus not satisfying, even less so if one notes that the time step is 5 times smaller than that based on the advective time. 


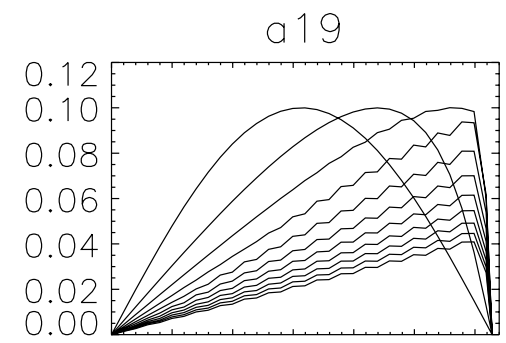

0.00 .51 .01 .52 .02 .53 .0

Fig. 3. Burgers equation: nonlinear viscosity $\left(\nu=5 \nu_{1}^{0}|\partial u / \partial x|\right)$

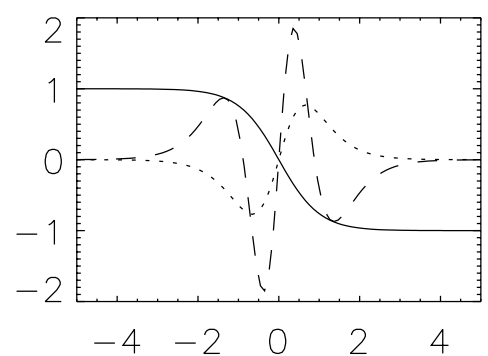

Fig. 4. Step function $(y=-\tanh x)$, its second (dotted line) and fourth derivative (dashed line) with negative sign

\subsection{Diagnostics and remedy}

Figure 2 shows, for three different filters, the damping rate of individual Fourier modes versus wave number, but gives no information on the damping of the quasi-discontinuous steps, for which the distribution of both the amplitude and phase with wave number is critical. Quasi-discontinuous steps appear commonly during the evolution of a turbulent flow. Specifically, we consider a step of the form $y=-\tanh x$. Figure 4 shows the function, its second derivative, and its fourth derivative multiplied by -1 . The last two profiles are proportional to the damping induced by a Laplacian and a bi-Laplacian term respectively, applied to the quasi-step function. The profile of the second derivative shows two oscillations of opposite signs: these oscillations allow the standard molecular dissipation terms to damp the left and right "wings" of the step function. In contrast, the bi-Laplacian term presents four oscillations: the two oscillations in the limited central region lead to damping but not the two lateral oscillations which amplify the signal, leading to the Gibbs effect.

This allows us to understand why all filters based on derivatives of order higher than the second derivative (or, in Fourier space, filters decreasing faster than a gaussian) generate parasitic, Gibbs-like oscillations. To reduce the oscillations generated by a non Gaussian filter, one is tempted to multiply it by a function which is non zero only in the central core: this is exactly what a viscosity proportional to the gradient does, as the one considered in the introduction to limit the action of the Laplacian.

The idea of a variable viscosity may actually be used for two purposes at the same time: to reduce the dissipation and to reduce the Gibbs effect. In the example of Fig. 3, the reduction of the Gibbs effect is obtained at

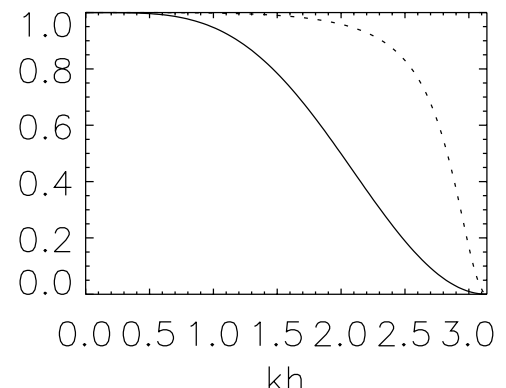

kh

Fig. 5. Two compact linear filters (Lele 1992) versus wave number $k$. The strong filter (continuous line) is used for Burgers equation, see Fig. 2. The weak filter (dotted line) is used for shear flows

the price of a too strong reduction of the dissipation. A possible compromise consists in extending the range of the viscosity, for example by considering a viscosity proportional to $|\partial u / \partial x|^{\alpha}$, with $\alpha$ smaller than unity. In the particular case of the bi-Laplacian, taking $\alpha=1 / 2$ could reduce the wings responsible for the Gibbs effect without too much reducing the dissipative core.

Inasmuch as the compact filter mentioned above gives results identical to those obtained by using a bi-Laplacian (Fig. 2, bottom), it is tempting, and it will prove to be useful, to apply the same ideas on a filter, be it only because the filter a priori requires no reduction of the time step. To apply the idea of non-uniform dissipation to a filter, we introduce the transformation:

$u->u+\epsilon(\hat{u}-u)$

where $\hat{u}$ is the filtered signal, and $\epsilon$ is proportional to $|\partial u / \partial x|^{\alpha}$, normalized by $\left(k_{\max } U\right)^{\alpha}$, so that $\epsilon$ is between 0 and 1 . This transformation will be defined in more detail below in Sects. 3 and 4, where it will be applied to the Burgers and shear flow problems.

\subsection{Numerical scheme}

The method used to compute the spatial derivatives (first and second) is one of the implicit (or compact) schemes studied extensively by Lele (1992). It is of 6 th order within the domain, 4th order at the boundaries. The interest of the implicit method lies in a strong reduction of the error, as compared to an explicit scheme of the same order, while it is at the same time faster than a spectral method.

Such a scheme is not dissipative in itself, hence one has to add an explicit diffusion term, or filter, to damp the excitation brought continuously at the grid scale by the nonlinear term. One can use either an explicit damping term, like the Laplacian or bi-Laplacian, or more generally a filter. The filters used here are defined implicitely, i.e., following the same method as for the spatial derivatives (Lele 1992). We use here in turn the two filters shown in Fig. 5, depending on the physical problem at hand. The "strong" filter, which damps most at the high frequencies, is used for the Burgers problem (it appeared already in Fig. 2). The other ("weak") filter is less dissipative, and 
is used for shear flows. Both filters are defined implicitely as follows:

$$
\begin{aligned}
& \alpha \hat{u}_{i-1}+\hat{u}_{i}+\alpha \hat{u}_{i+1}=a u_{i}+b\left(u_{i+1}+u_{i-1}\right) \\
& +c\left(u_{i+2}+u_{i-2}\right)+d\left(u_{i+3}+u_{i-3}\right) .
\end{aligned}
$$

The weak filter satisfies the following relations (a particular case of Eq. (C.2.4) of Lele 1992):

$$
\begin{array}{ll}
a=(5+6 \alpha) / 8 & b=(1+2 \alpha) / 4 \\
c=-(1-2 \alpha) / 16 & d=0 .
\end{array}
$$

To obtain the sixth-order, and also the lowest dissipation as well, we take $\alpha=0.475$ in Eq. (8). The strong filter satisfies to (Eq. (C.2.6) of Lele 1992):

$$
\begin{array}{ll}
a=(2+3 \alpha) / 4 & b=(9+16 \alpha) / 32 \\
c=\alpha / 8 & d=-1 / 32
\end{array}
$$

with $\alpha=0.49$. Note that at the boundaries an explicit, non-centered, fourth-order filter is used (Eq. (C.2.11) of Lele 1992).

The temporal scheme is second order Euler (for the Burgers equation) and third-order Runge-Kutta for the Euler equations. For the Burgers equation, the time step is constant and equal to the smallest of the initial advective $\left(1 /\left(k_{\max } U\right)\right)$ and dissipative times. For the Euler equations, the time step is variable, and based on the smallest of the dissipative and advective times $\left(1 / k_{\max }\left(|u|_{\max }+\right.\right.$ $c)$ )), where $c$ is the sound speed.

In the following, the Burgers equation is integrated in the domain $[0,2 \pi]$, with $N=64$ grid points, except for run a28 for which $N=256$ (see Table 1 ). The velocity is fixed to zero at the boundaries.

The Euler equations are written in terms of spherical coordinates $(r, \theta, \phi)$. Axisymmetry is assumed (no dependence on $\phi$ ). The domain is bounded by two spherical shells $r^{0} \leq r \leq r^{1}$ and also in latitude, $\pi / 2-\pi / 8 \leq \theta \leq$ $\pi / 2+\pi / 8$, symmetric around the equator $(\theta=\pi / 2)$. No conditions are imposed at the outer boundary since the flow is supersonic there. At the inner shell, the boundary conditions are imposed via the characteristics entering the domain: the temperature is fixed (if the radial flow velocity is positive), the entering sound wave flux is set to zero (cf. Grappin et al. 1997). The characteristics method has been shown to be well adapted to the fluid equations even in the presence of dissipative terms (Poinsot \& Lele 1992). A last characteristic has to be imposed, which is related to the transverse velocity: this is detailed in Sect. 4 . The various boundary conditions for the shear flows are listed in Table 2.

\section{Nonlinear filters for the shock formation problem (Burgers)}

We consider the problem of defining an optimal filtering scheme for shock formation. We have seen in the introduction that one could suppress the Gibbs effect by considering a Laplacian with a viscosity varying as the gradient of the velocity, but that a noise appeared at the
Table 1. Characteristics of runs for the Burgers equation and the shear flow instability. The first letter of the shear flow runs denotes the boundary condition (see Table 2). NLF means nonlinear filter (see Eqs. (11) and (12), respectively for the Burgers and the shear flow problems). LF denotes the weak linear filter (Eq. (8)). "Dissip" denotes ordinary viscous runs. Resolution is $N=64$ for all Burgers runs except a28, and 540 by 180 for the shear flows. Except for run A2, all shear flow runs have the same linear (weak) filter for all degrees of freedom except temperature. Only the filtering of temperature is varied from one run to the other

\begin{tabular}{ccc}
\hline Run & Scheme & Results \\
\hline & Burgers \\
a18a & $\nu=1.5 \nu^{0}$ & dissip. \\
a16 & Bi-Laplacian $\nu_{4}=4 \nu_{4}^{0}$ & Gibbs \\
a19 & $\nu=5 \nu_{1}^{0}|\partial u / \partial x|$ & noise \\
a20 & $\nu=0.2 \nu^{0}+5 \nu_{1}^{0}|\partial u / \partial x|$ & small $d t$ \\
a28 & $\nu=1.5 \nu^{0}(N=256)$ & dissip. \\
a25d & $\mathrm{NLF}, \nu=0.3 \nu^{0}$ & Gibbs \\
a25e & Double NLF $\nu=0.3 \nu^{0}$ & OK \\
a29a & $\mathrm{NLF}, \nu=0.2 \nu^{0}+\nu_{1 / 2}^{0}|\partial u / \partial x|^{1 / 2}$ & OK \\
& & \\
& $\mathrm{Shear}$ flows \\
A1 & $\mathrm{LF}$ & Gibbs \\
A2 & $\nu_{\alpha \beta}=\nu_{1}^{0}\left|\partial u_{\alpha} / \partial \beta\right|$ & Gibbs \\
B1 & $\mathrm{LF}$ & Gibbs \\
B2 & $\mathrm{NLF}$ & $\simeq$ Gibbs \\
C1 & $\mathrm{NLF}$ & Gibbs \\
C2 & $\mathrm{NLF}, \kappa=0.5 \kappa^{0}$ & OK \\
D1 & $\mathrm{LF}$ & Gibbs \\
D2 & $\mathrm{NLF}, \kappa=0.25 \kappa^{0}$ & OK \\
D3 & $\mathrm{NLF}, \kappa=0.1 \kappa_{1 / 2}^{0}\left|\partial T / \partial x_{\alpha}\right|^{1 / 2}$ & $\simeq$ OK \\
\hline
\end{tabular}

Table 2. Boundary conditions for shear flows. The $u_{\theta}$ profile is characterized by its shape and amplitude. "Asymmetric" as the $u_{\theta}$ profile in Fig. 9 (top). "Symmetric" denotes a profile which is an even function of latitude. "Small" means the same amplitude as in Fig. 9; "large" means twice that amplitude

\begin{tabular}{ccc}
\hline B.C. & Temperature & $u_{\theta}$ \\
\hline $\mathrm{A}$ & {$[1,1.5]$} & small, asymmetric \\
$\mathrm{B}$ & {$[1,1.5]$} & large, asymmetric \\
$\mathrm{C}$ & {$[.9,1.4]$} & large, asymmetric \\
$\mathrm{D}$ & {$[.9,1.4]$} & symmetric \\
\hline
\end{tabular}

grid scale. To suppress this noise, we can add a standard viscous term. If the noise disappears using a viscosity smaller than the minimal value obtained below for the standard Burgers equation $\left(\nu=1.5 \nu^{0}\right)$ then we will have made some progress. Figure 6 shows that using $\nu=0.2 \nu^{0}+5 \nu_{1}^{0}|\partial u / \partial x|$ is acceptable (the solution with standard viscosity at higher resolution, $N=256$, is shown for comparison). But the price is a strong reduction of the time step, because of the factor 5 in front of $\nu_{1}^{0}$ (see introduction above). 


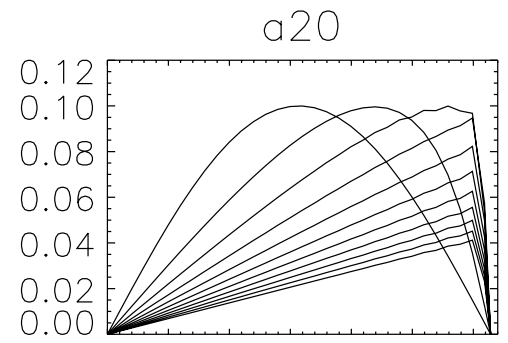

0.00 .51 .01 .52 .02 .53 .0

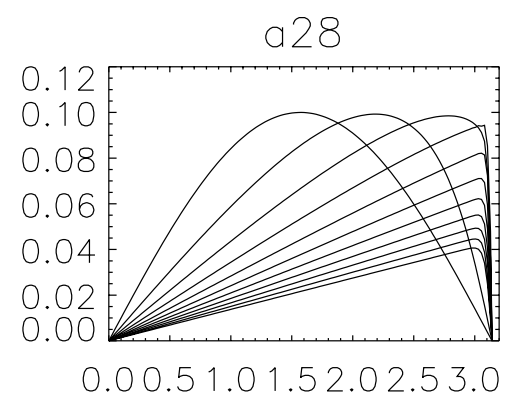

Fig. 6. A (costly) compromise for Burgers equation: nonlinear plus linear viscosity $\left(\nu=0.2 \nu^{0}+5 \nu_{1}^{0}|\partial u / \partial x|\right.$, top $)$, compared with a higher resolution run $(N=256)$ with standard damping $\nu=1.5 \nu^{0}$ (bottom)

It is possible to get rid of any classical viscous term (hence reducing more the damping of large scales) if one uses a viscosity proportional, not to the gradient, but to the square root of the gradient. A satisfying result can be obtained with $\nu=5 \nu_{1 / 2}^{0}|\partial u / \partial x|^{1 / 2}$. Again, however, the high value of the prefactor $\left(5 \nu_{1 / 2}^{0}\right)$ forces one to reduce strongly the time step. It is thus interesting to examine whether the filter, which does not require us to reduce the time step, may be transformed in the same way.

We thus replace the explicit diffusive term by the strong filter shown in Fig. 5. As previously with the biLaplacian, one finds that the numerical noise is now absent, while a Gibbs effect appears, which is $10 \%$ of the total amplitude. In order to reduce the area subjected to the Gibbs effect, we add some (small) viscous damping: viscosity is $\nu=0.2 \nu^{0}$. However, this does not reduce the amplitude of the Gibbs effect generated by the filter. Also, calling the filter several times per time step (2 or 4 times) does not change anything. A complete reduction of the Gibbs effect requires taking a large (standard) viscosity, $\nu=\nu^{0}$, which is no significant progress in terms of dissipation reduction, compared to the standard value (with no filter) of $\nu=1.5 \nu^{0}$.

In order to reduce the Gibbs effect generated by the filter, we use the transformation defined in Eq. (6). We begin by using a prefactor proportional to the gradient:

$u->u+\epsilon_{1}^{0}|\partial u / \partial x|(\hat{u}-u)$

with $\epsilon_{1}^{0}=1 /\left(k_{\max } U\right)$. The Gibbs effect is indeed reduced, but a numerical noise shows up everywhere, making the computation useless. This means that we reduce too much the area where dissipation is active. Adding some viscos-

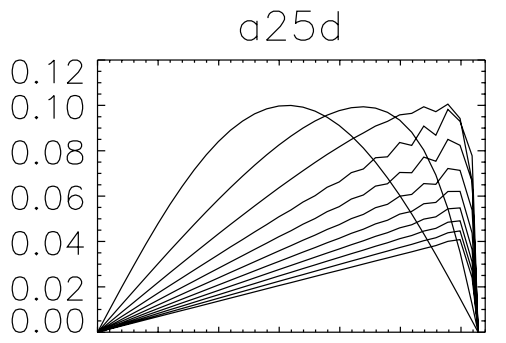

0.00 .51 .01 .52 .02 .53 .0

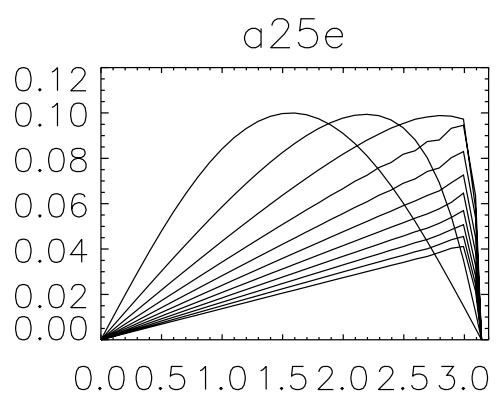

Fig. 7. Burgers equation, nonlinear filter (Eq. (11)) plus some viscosity $\left(\nu=0.3 \nu^{0}\right)$. Top: filter called once per time step; bottom: filter called twice per time step

ity $\left(\nu=0.2 \nu^{0}\right)$ does not really improve the result. We thus consider a intermediate solution, with a prefactor proportional to the square root of the gradient:

$u->u+\epsilon_{1 / 2}^{0}|\partial u / \partial x|^{1 / 2}(\hat{u}-u)$

with $\epsilon_{1 / 2}^{0}=1 /\left(k_{\max } U\right)^{1 / 2}$. As a result, the noise is still very important. If we add a viscosity $\nu=0.3 \nu^{0}$ to the filter, the noise is still present, and the Gibbs effect as well, although somewhat reduced (Fig. 7, top). But, if one calls the filter twice per time step, the Gibbs effect is almost eliminated (Fig. 7, bottom). This method is very sensitive to the precise choice of parameters: if one calls the filter 4 times per time step, then the Gibbs effect reappears. A result equivalent to that of Fig. 7 (bottom) may be obtained with just one call per time step if one decreases the ordinary viscosity but adds a viscosity proportional to the square root of the gradient: $\nu=0.2 \nu^{0}+\nu_{1 / 2}^{0}|\partial u / \partial x|^{1 / 2}$ (Fig. 8). Contrary to the solution shown in Fig. 7 (bottom), this last solution does not require one to decrease the time step below the advective value, and the dissipation is minimum.

We do not seek here further improvement or refinement of the method, such as optimizing the parameters, because our aim is the study of shear flows, which, as we see now, requires a specific adaptation of the method.

\section{Turbulent mixing of shear flows}

We apply now the method developed above to shear flows in the accelerating region of a non-magnetic solar wind. Note that because the problem is different, a direct application of the method will a priori not work well. Compared to the Burgers problem, the shear flow we consider now 


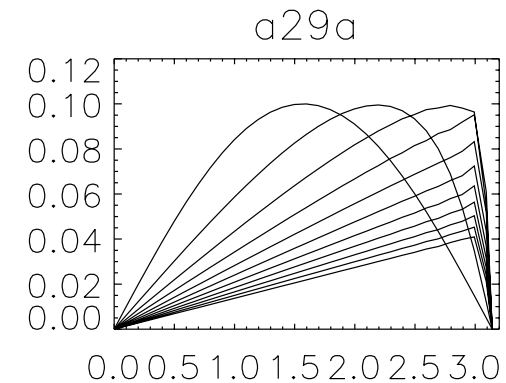

Fig. 8. Burgers equation, nonlinear filter plus nonlinear viscosity $\left(\nu=0.2 \nu^{0}+\nu_{1 / 2}^{0}|\partial u / \partial x|^{1 / 2}\right)$

has more degrees of freedom, and, since the flow is $2 \mathrm{D}$, the compressive effects should be reduced. The main phenomenon is now the advection and steepening of thermal gradients induced by the instability of the interface between cold and hot streams. In fact, the strong filter appears to be unnecessarily strong for the present problem. We consider thus as our starting filter the weak filter of Eq. (8).

The coordinates are the distance to Sun $r$, and colatitude $\theta$. We integrate the Euler (or Navier-Stokes) equations with a central gravitational field proportional to $1 / r^{2}$. We assume axisymmetry, and a polytropic index unity, which implies that the thermal inhomogeneities are advected with no variation of temperature (Grappin et al. 1997). With these conditions, a static atmosphere is unstable. When the inner boundary (that we will call "the corona") has a uniform temperature, a stable spherically symmetric flow, namely Parker's transonic solution, develops from the sun towards the exterior. This flow reaches the sound speed $c=\sqrt{T}$ at a distance $R^{\star}=G M /(2 T)=$ $1 /(2 T)$.

If the corona contains thermal inhomogeneities, then the cold coronal regions are the source of slow flows, while the hot regions are sources of fast flows. We consider here the particular case of a cold equatorial region with two hot regions surrounding it. The cold region $\left(T=T_{a}\right)$ generates a wind which reaches the speed of sound at about $R_{a}^{\star}=$ $1 /\left(2 T_{a}\right)$, while the hot regions $\left(T=T_{b}\right)$ generate a faster wind with a sonic point at about $R_{b}^{\star}=1 /\left(2 T_{b}\right)$. When the temperature difference is large enough, the boundary between the two flows becomes unstable, and a turbulent wake can develop.

The domain we consider is between $r=0.25$ and 1.21 , the unit distance is twice the sonic point associated with temperature $T=1$ (in other words, $R^{\star}=1 /(2 T)$ ). The coronal temperature is such that the sonic surface is within the numerical domain at all latitudes. The distance interval considered corresponds to about 5 to 24 solar radii. The wake instability depends on three parameters: the distance to the corona (where temperature is fixed), the temperature profile, and finally the amplitude of the transverse velocity $u_{\theta}$.

At the outer boundary, the flow is supersonic, so that no condition is imposed. The inner boundary conditions

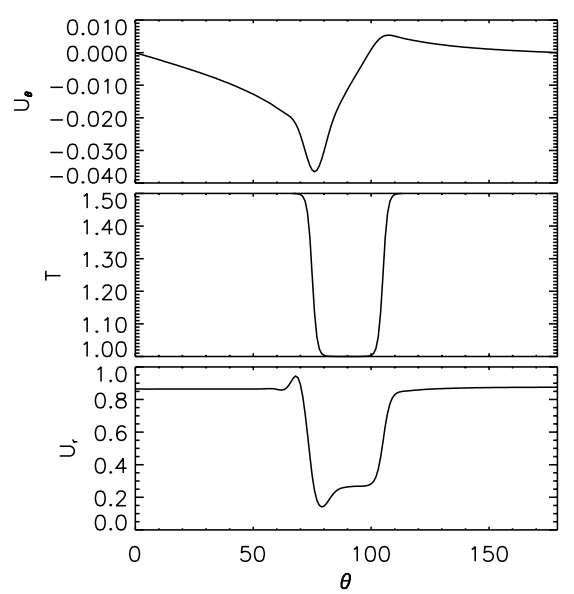

Fig. 9. Cold wake in a stellar wind, inner boundary conditions for run A1. From top to bottom: transverse velocity $u_{\theta}$, temperature and radial velocity versus latitude. Note that only temperature and $u_{\theta}$ are fixed, while the radial velocity is an outcome of the calculation. The sound speed is $\sqrt{T}$, hence is between 1 and 1.2

are as follows (Table 2): one imposes the temperature profile, and a zero flux of incoming acoustic waves. As a consequence, the density profile ajusts itself so that the pressure is uniform. A last condition is related to the transverse velocity; either (a) the transverse velocity $u_{\theta}$ is fixed, or (b) its radial derivative is zero. In case (a) ( $\mathrm{A}, \mathrm{B}$ and $\mathrm{C}$ in Table 2), the profile adopted for the transverse temperature is asymmetric with respect to the equator, which is the center latitude of the cold region (see Fig. 9). This favours the sinuous (S) modes, which are the most unstable. Two different amplitudes are considered. Case (b), corresponding to condition D in Table 2, suppresses any $\mathrm{S}$ modes, which a priori increases the stability of the flow. The other parameter which is varied is the mean temperature, which takes two values, as the temperature profile is characterized by its minimum and maximum temperatures, either $[1,1.5]$, or $[.9,1.4]$. Varying the temperature changes the stability of the flow, because lowering the temperature pushes the sonic line towards larger distances, so that at a given distance the mean divergence of the velocity decreases, which leaves the shear instability free to develop (Grappin et al. 1997).

Figure 9 shows profiles at the bottom boundary for run A1. The figure shows $u_{\theta}$, the temperature and the radial velocity. Since the transverse velocity profile is not symmetric with respect to the symmetry axis of the cold stream originating from the cold inner boundary region, S-waves are excited. Temperature varies at the inner boundary from 1 to 1.5 , and the transverse velocity $u_{\theta}$ from -0.04 to 0.01 . These two profiles are imposed. Recall that the radial velocity profile is free; its oscillations visible in the figure are physical; they correspond to rapid variations in the divergence of flowlines with latitude. The sound speed is $c=\sqrt{T}$, which is in between $[1,1.2]$; hence the Mach number at the inner boundary is between 0.2 and 0.9 . 


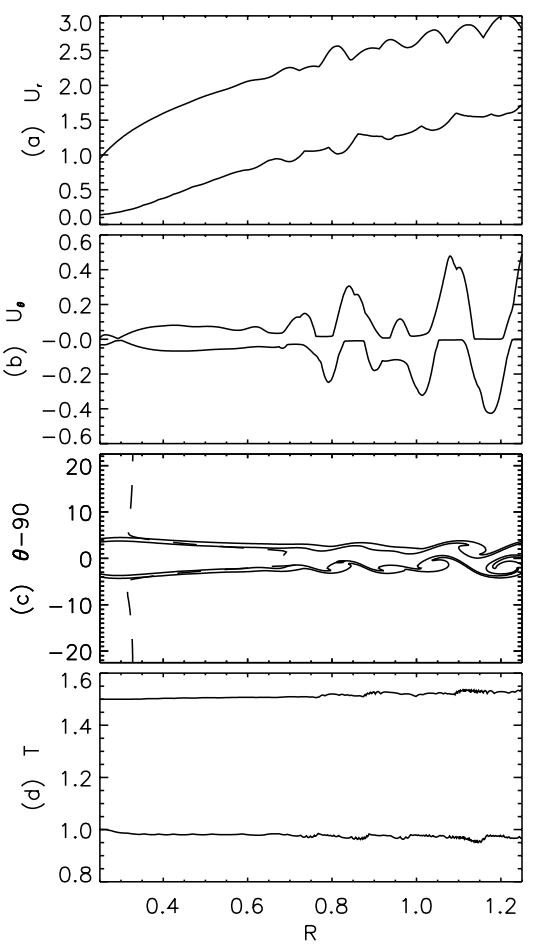

Fig. 10. Snapshot of radial variations in the wake problem (run A1): a) Extrema of radial velocity $u_{\mathrm{r}}$ versus heliocentric distance $R ; \mathbf{b})$ Extrema of transverse velocity $u_{\theta}$ versus $R ; \mathbf{c}$ ) Temperature contours $(1.1,1.4)$ in the $R-\theta$ plane; the cold region lies around the equator; the dashed line indicates the sonic line with $\left.u_{\mathrm{r}}=c_{\mathrm{s}} ; \mathbf{d}\right)$ Temperature extrema versus $R$

Resolution in $R, \theta$ is of $540 * 180$ grid points. If the integration is made using a classical viscosity adapted to the resolution $\left(\nu \simeq \nu^{0}=U / k_{\max }\right.$, where $k_{\max }=$ $\sup (\delta r / \pi, r \delta \theta / \pi))$, then the flow is laminar, and the transverse velocity decreases with distance approximatively as $1 / r$, which amounts to conserving angular momentum during advection. One expects that at high enough Reynolds number, the interface between the slow flow generated by the cold region and the fast flow becomes unstable (Kelvin-Helmholtz instability). On the other hand, we know that the expansion (divergence) of the global flow is an inhibiting factor: in order for the flow to be unstable, the usual instability rate must be higher than the divergence of the mean velocity (Grappin 1996; Grappin et al. 1997).

\subsection{Large mean temperature, small $u_{\theta}(A)$ - linear filter versus nonlinear viscosity}

Figure 10 shows a snapshot of the flow properties versus distance to sun, once the flow has reached a quasistationary regime (run A1). We use the weak, linear filter defined in Sect. 2 (Eq. (8)). Figure 10 shows the radial variation of the extrema of the radial velocity, transverse velocity $u_{\theta}$, and temperature, as well as temperature contours in the $r-\theta$ plane. The extrema are found by computing the maximum and minimum over all latitudes at a given distance: for instance, for temperature the maximum indicates the temperature outside the cold stream, and the minimum corresponds to the temperature in the middle of the cold stream. Because the polytropic index is unity, the minimum temperature versus heliocentric distance should be either a constant, or increasing with distance, if some diffusion takes place. Also, the maximum temperature should be either a constant, or decreasing; any deviations from these rules are a signature of the Gibbs effect. One sees that the velocity remains very different in the cold and hot flows (Fig. 10a). The instability of the interface between the cold and hot wind is revealed by the growth of transverse velocity extrema with distance (Fig. 10b). The interface itself is visualized by the temperature contours, which show the formation of whirls. Temperature in cold and hot flows is shown by the temperature extrema versus radial distance in Fig. 10d. One sees an unphysical increase of the maximal temperature and a decrease of the minimum temperature, revealing a strong Gibbs effect which starts very close to the inner boundary. On the other hand, there is no large-scale mixing of the temperature, which would appear as a marked increase in the minimum temperature; mixing would necessitate pushing the two temperature interfaces against each other, i.e., strongly pinching the cold flow, which does not occur here.

As a first attempt to reduce the Gibbs effect, we replace the linear filter for velocity and temperature by a Laplacian with variable coefficients $\nu_{\alpha \beta}$, proportional to the gradients of the fields. We consider for the diffusive term associated with the component $\alpha(r$ or $\theta)$ of the velocity: $\nu_{\alpha r} \partial^{2} u_{\alpha} / \partial r^{2}+\nu_{\alpha \theta} \partial^{2} u_{\alpha} / \partial \theta^{2}$, with $\nu_{\alpha_{r}}=$ $\nu_{1}^{0}\left|\partial u_{\alpha} / \partial r\right|, \nu_{\alpha \theta}=\beta \nu_{1}^{0}\left|\partial u_{\alpha} / \partial \theta\right|$, where $\nu_{1}^{0}=1 / k_{\max }^{2}$, and $\beta$ taking into account the aspect ratio of the $R-\theta$ mesh. A similar term is used for temperature. This choice leaves the time step unchanged, i.e., based on the advective time.

The result is shown in Fig. 11. The interface instability starts somewhat closer to the inner boundary than previously with run A1 (Fig. 10), but the Gibbs effect is much larger now than in the previous case. Recall that when using nonlinear viscosity with the Burgers equation, even with a viscosity five times the critical value, we still obtained substantial noise at the grid scale (Fig. 3).

\subsection{Large mean temperature, large $u_{\theta}(B)$ - nonlinear filtering}

Following the same steps as in the Burgers case, we now modify the filter. Since we observed that the Gibbs effect mainly affects the temperature field, we modify only the temperature filter. For the other fields, we use the linear filter already used for run A1 (Fig. 10). Let us consider first filtering the temperature as follows (cf. Eq. (11)):

$$
\begin{array}{r}
T->T+\epsilon(\hat{T}-T) \\
\epsilon=|\partial T / \partial r|^{1 / 2} /\left(k_{\max }^{r} \delta T\right)^{1 / 2}
\end{array}
$$




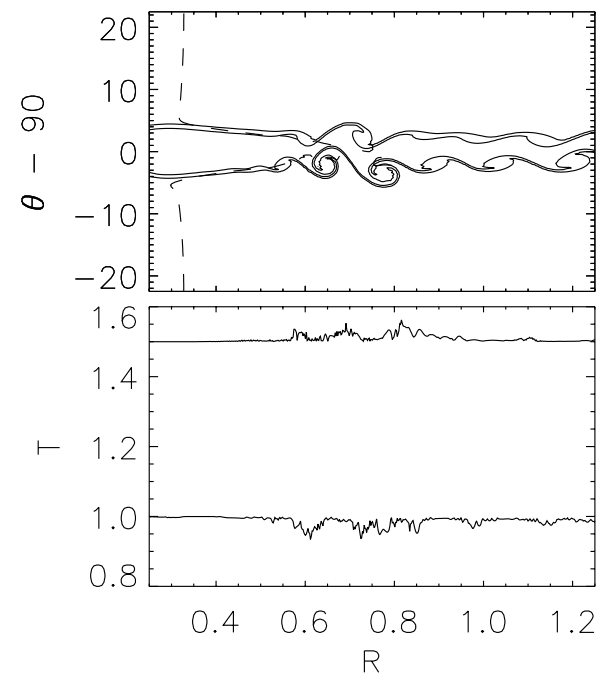

Fig. 11. Wake (run A2) with nonlinear diffusive terms $\left(\nu_{\alpha \beta}=\right.$ $\nu_{1}^{0}\left|\partial u_{\alpha} / \partial \beta\right|$, compare Figs. 10c-d). Top: Temperature contours in the $R-\theta$ plane; bottom: Temperature extrema versus radial distance

where $k_{\max }^{\mathrm{r}}=\pi / \Delta r, \Delta r$ being the radial mesh size and $\hat{T}$ the temperature field filtered in the radial direction. We proceed in the same way for filtering in the $\theta$ direction. The quantity $\delta T$ is defined so that $\epsilon$ is everywhere smaller than unity; a convenient choice is $\delta T=\left(T_{\max }-T_{\min }\right) / 2$, which is here 0.25 .

Figure 12 compares the results obtained by using successively the linear filter (Figs. 12a and b) and the nonlinear filter (Figs. 12c and d) for the temperature. We have increased the transverse velocity by a factor two (condition B), in order to more trigger the instability. One sees indeed by comparing Fig. 12(top) and Fig. 10 that the increase in the transverse velocity (using the same linear filter) greatly increases the instability of the flow: the instability sets in much closer to the inner boundary and turbulent mixing appears, as shown by the increase of the minimal temperature in the second part of the domain. However, we see that this is achieved at the price of a much increased Gibbs effect, especially close to the inner boundary. As a consequence, it is difficult to trust even the qualitative flow pattern. In fact, the instability is probably initiated by a whirl which forms very close to the boundary, and which raises locally the temperature gradient. This is the source of a strong Gibbs oscillation close to the boundary, which in turn probably increases the whirl amplitude.

Using the nonlinear filter (Figs. 12c and d) gives strinkingly different results. The Gibbs effect is much reduced, but, at the same time, the instability is much weakened, as it appears only close to the outer boundary. Hence, it seems difficult to obtain a strongly unstable flow devoid of Gibbs effect.

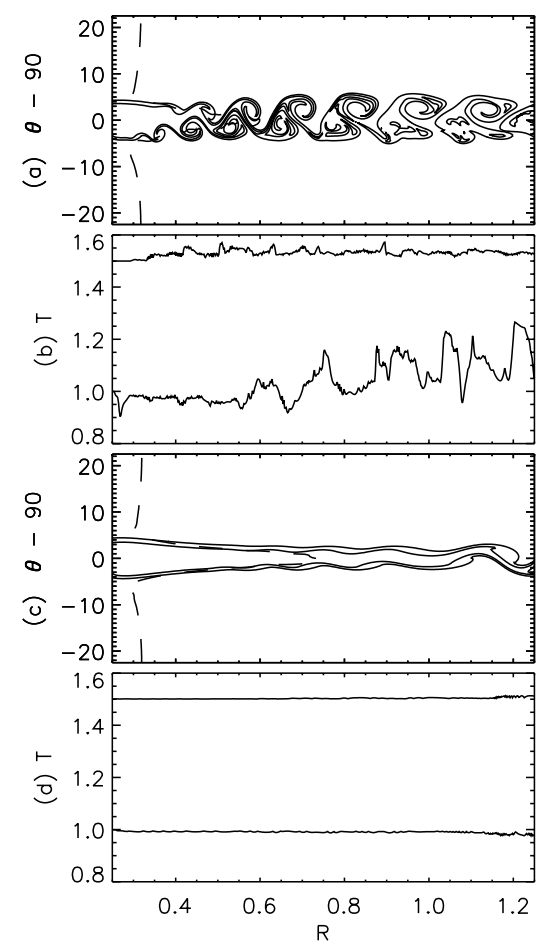

Fig. 12. Wake with large transverse velocity, comparison of linear and nonlinear filtering. Top (a-b), run B1), linear filter for the temperature. Bottom (c-d), run B2), nonlinear filter for the temperature. a) and c) Temperature contours $(1.1,1.4)$ in the $R-\theta$ plane. b) and $\mathbf{d}$ ) Temperature extrema versus $R$

\subsection{Decreasing the mean temperature (C) - nonlinear filtering plus small conductivity}

The previous results strongly suggest that the reduction of the Gibbs effect obtained via the nonlinear filter is largely related to the stabilization of the shear instability brought by the lower effective Reynolds of the flow associated with the nonlinear filtering scheme.

One might thus ask whether the Gibbs effect would reappear if one would again (using the same numerical filter) increase the physical instability of the flow. In order to examine this point, we could again increase the transverse velocity at the inner boundary, but we prefer to consider now the effect of decreasing the temperature.

Decreasing the temperature should decrease the average flow speed, hence leaving more time for the instability to grow. We fix the new temperature range to be between 0.9 and 1.4 (condition C, see Table 2). Figure 13 shows the result obtained with the nonlinear filter. One sees that the relative position of the sonic line remains mostly unchanged when compared to the previous runs, but nevertheless, it is clear that the whole flow has become much more unstable (compare with run B2, Figs. 12c and $\mathrm{d}$, same nonlinear filter). The reason lies in the detailed velocity profile as we shall see in the discussion (see below Fig. 18).

Both the instability onset and the turbulence mixing occurs now much closer to the inner boundary than in the 


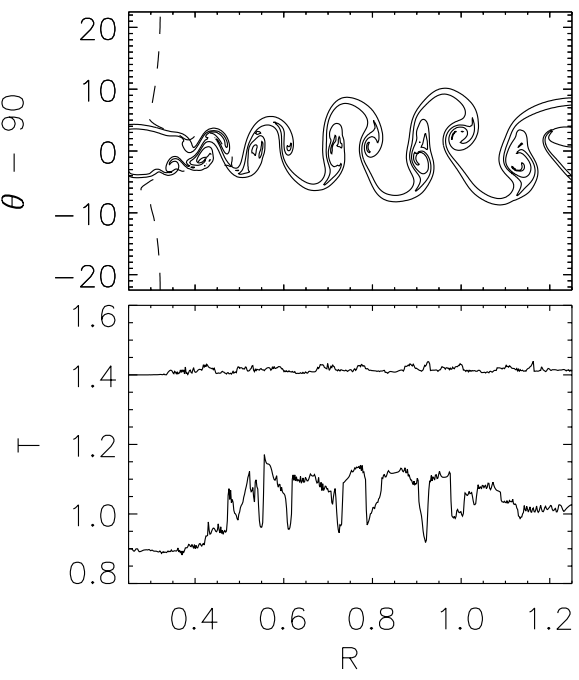

Fig. 13. Wake with low mean temperature (run C1), nonlinear filter for the temperature. Top: Temperature contours $(1,1.3)$ in the $R-\theta$ plane; bottom: temperature extrema versus $R$

previous run (compare with Figs. 12c-d). However, our pessimistic hypothesis is seen to be correct: the Gibbs effect is present again, rather close to the inner boundary, although not so strong as previously with the linear filter (run B1, Figs. 12a-b). One can thus not exclude the hypothesis that the Gibbs effect plays a role in the onset of the observed instability itself.

To investigate this point, we completely eliminate the Gibbs effect by adding a Laplacian term in the temperature equation, corresponding to a classical conductivity $\kappa$. Figure 14 shows the evolution with a conductivity half the critical value, $\kappa=\kappa^{0} / 2$, where the critical value is defined as:

$\kappa^{0}=0.5\left(T_{\max }-T_{\min }\right) / k_{\max }$.

Surprinsingly enough, the Gibbs effect has completely disappeared, but the vortex street is still present, and moreover not so different from the preceding case; the instability and the turbulent mixing set in close to the inner boundary. Varying the value of the conductivity (for instance taking $\kappa=0.25 \kappa^{0}$ ), or considering a conductivity varying as the square root of the temperature gradient: $\kappa=\kappa_{1 / 2}^{0}|\partial T / \partial r|^{1 / 2}$, we obtain only slight variations with respect to the preceding result.

\subsection{Low mean temperature, symmetric $u_{\theta}(D)$ - further reduction of dissipation}

We now change qualitatively the boundary conditions, considering a symmetric profile of the transverse velocity at the boundary. This is achieved by imposing a zero radial derivative of $u_{\theta}$ at the inner boundary. In this way, the profile is an odd function of the latitude, i.e., of the distance to the center of the cold region. The temperature is unchanged (condition D, see Table 2). The cold wake should be more stable in this case, as the sinuous

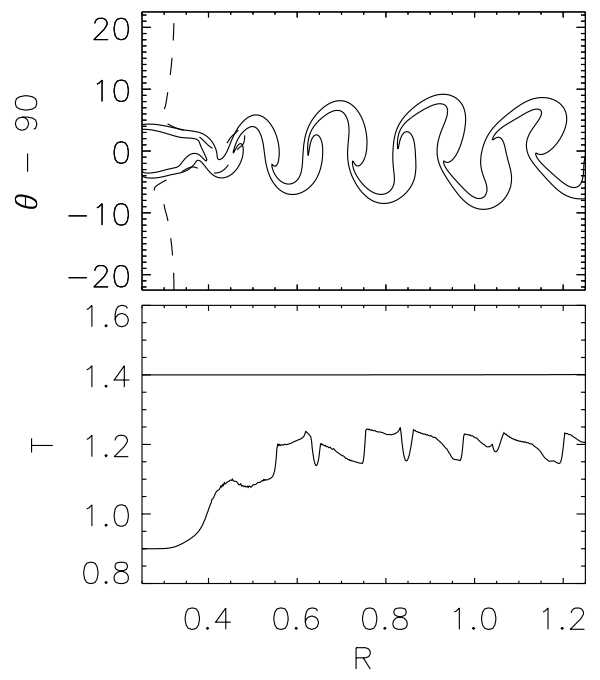

Fig. 14. Wake with low mean temperature (run C2), nonlinear filtering for temperature plus a small conductivity $\left(\kappa=0.5 \kappa^{0}\right)$. Same caption as in Fig. 13

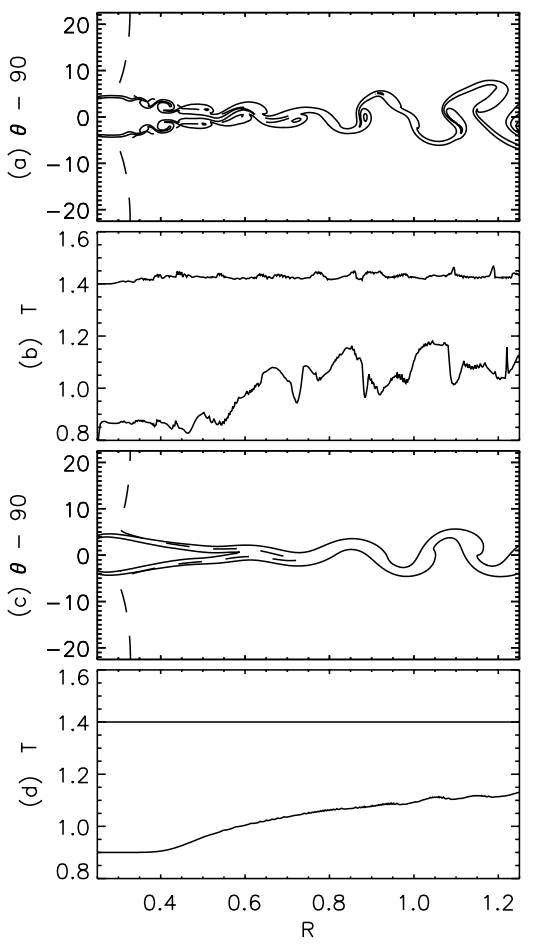

Fig. 15. Wake with symmetric $u_{\theta}$. Top (run D1, a-b)): linear filter. Bottom (run D2, c-d)): nonlinear filter with weak conductivity $\kappa=0.25 \kappa^{0}$. a) and c) Temperature contours $(1,1.3)$ in the $R-\theta$ plane. b) and $\mathbf{d}$ ) Temperature extrema versus $R$

modes are in principle no longer excited close to the inner boundary.

Figure 15 allows us to compare the results obtained using respectively the linear filter and the nonlinear filter depending on the square root of the gradient, with a conductivity $\kappa=0.25 \kappa^{0}$. The S-modes are indeed absent close to the inner boundary, but they are seen to dominate at larger distances, due to numerical errors providing seeds for S-modes, and to the larger growth rate of S-modes. 


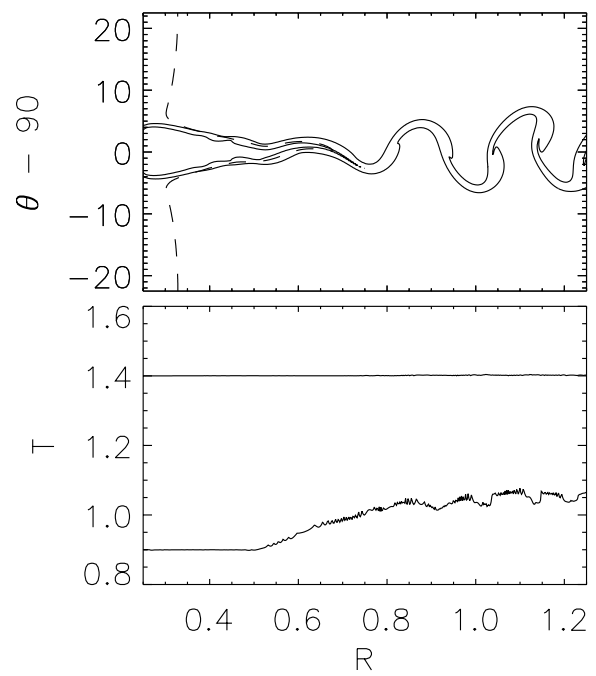

Fig. 16. Wake with symmetric $u_{\theta}$ (run D3), nonlinear filter with weak nonlinear conductivity $\left(\kappa=0.1 \kappa_{1 / 2}^{0}|\partial T / \partial r|^{1 / 2}\right)$. Top: Temperature contours $[1,1.3]$ in the $R, \theta$ plane; bottom: Extrema of temperature versus $R$

The comparison of both runs clearly shows the loss of information caused by the non-zero conductivity: all details below 10 mesh points are lost in run D2; however, the distance for turbulent mixing onset is about the same in both runs. Besides, the Gibbs effect, which was with the linear filter very strong close to the inner boundary, and thus probably played a role in the instability onset, is completely absent in the case of run D2 (Fig. 15d).

As with the previous boundary conditions (C), it is possible to push still further the reduction of dissipation. For this purpose, we replace the classical conductivity with a conductivity varying with the square root of the temperature gradient: $\kappa=0.1 \kappa_{1 / 2}^{0}$. Figure 16 shows that some small structures in the cold wake which were visible when using the linear filter reappear (compare Fig. 15, top). In particular, the longer length of the thin cold "tong" corresponds to a larger distance for the onset of mixing. The Gibbs effect does not appear before the onset of instability, but some noise is visible in the minimum temperature profile, at distances larger than the onset of mixing. Figure 17 summarizes the radial profiles of transverse velocity extrema for the three runs (D1, D2, D3) with symmetric transverse velocity: with linear filter, nonlinear filter with standard conductivity, nonlinear filter with conductivity varying as the square root of the gradient.

\section{Discussion}

We considered the problem of the turbulent mixing, in non-magnetic stellar winds, of streams induced by timeindependent thermal inhomogeneities in the corona. For that purpose, we developed a filtering scheme able to increase the effective Reynolds number without additional computer cost, and without generating the spurious Gibbs oscillations which develop usually close to sharp variations

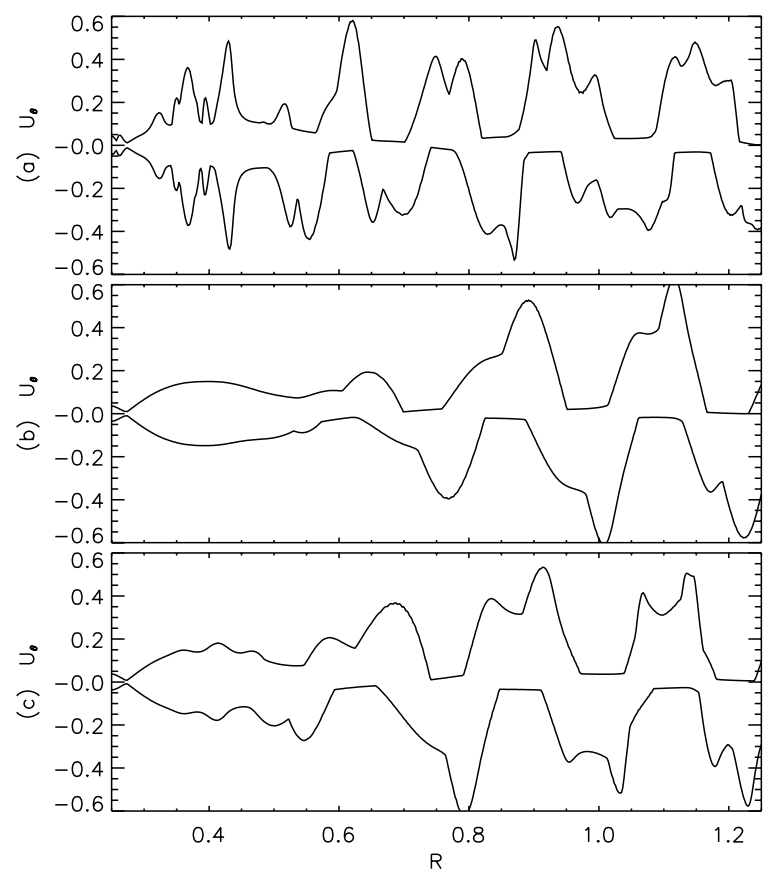

Fig. 17. Comparison of transverse velocity profiles (minimum and maximum) with symmetric $u_{\theta}$. From top to bottom: a) run D1, linear filter b) run D2, nonlinear filter with some conductivity c) run D3, nonlinear filter with nonlinear conductivity

(shocks or vorticity sheets) when using non Gaussian filters, i.e., hyperviscosities.

We first tested the filtering scheme by considering the Burgers equation, which models the shock formation. We then considered the problem of unstable cold wakes in stellar winds. We varied the filtering techniques to find the optimal scheme. The nonlinear filter we propose (see, in Table 1, runs marked with OK), is to be combined with some (small) non-linear or linear conductivity; this eliminates Gibbs oscillations with no additional computational cost. Varying the amount of reduced conductivity showed that the method is convergent (the instability rate does not change). In contrast, using filtering with no caution leads to artificially unstable flows, the spurious noise introduced by the filtering itself triggering the instability. The properties of our filtering scheme are in principle shared by other schemes proposed by Passot \& Pouquet (1988), Siregar et al. (1995), Guermond (2000), but our scheme should be less demanding in computer time.

The parameters considered here are not unreasonable compared to coronal standards. The values adopted for the transverse velocity seed correspond to a transverse Mach number $u_{\theta} / c$ between 0.04 and 0.08 . The temperature fluctuations considered were about $50 \%$, comparable to that observed in the distant, supersonic solar wind. The conclusion thus seems to be that stream instabilities in the accelerating region should be a natural byproduct of coronal turbulence, if the corona were devoid of magnetic fields.

The results of the different runs (except the last one) are summarized in Fig. 18, which gives a snapshot of the 

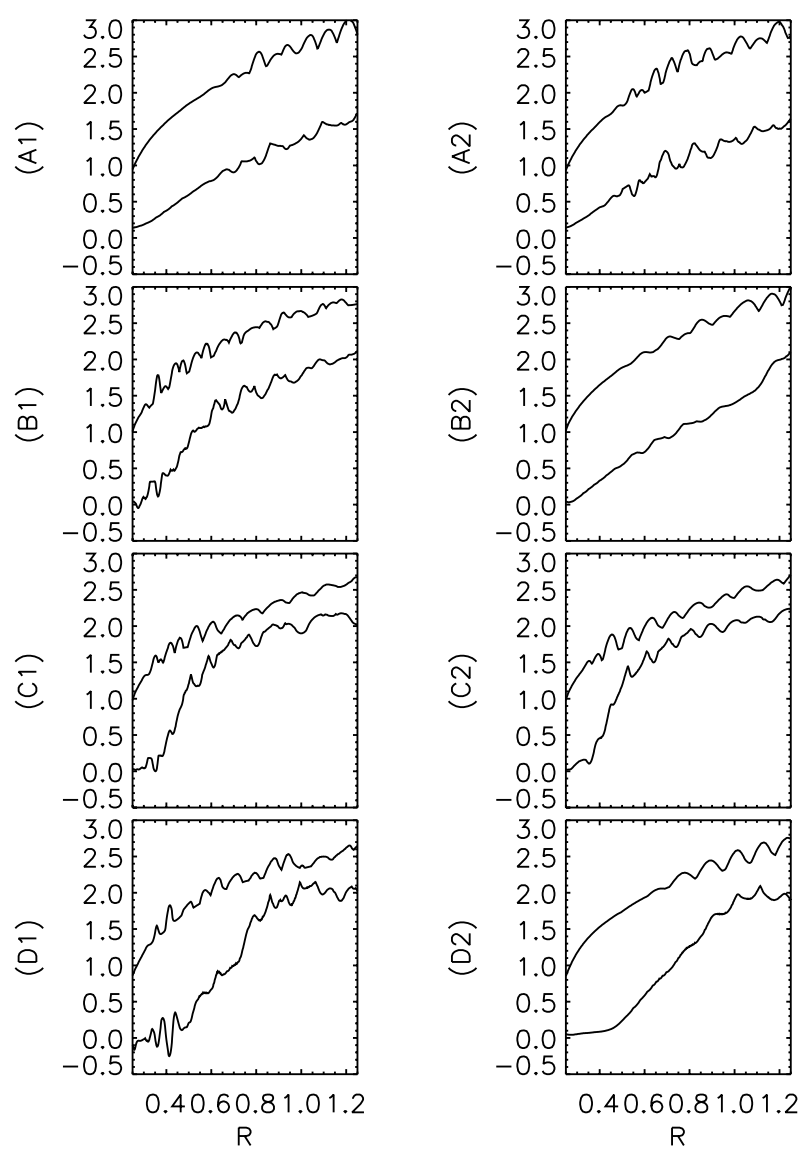

Fig. 18. Extrema of radial velocity, snapshots of all shear flow runs, except the last one (see Tables 1 and 2). From top to bottom, boundary conditions A to $\mathrm{D}$

radial profiles of the minimum and maximum radial velocity. Each row corresponds to a different boundary condition: from top to bottom, one shows conditions A to D. Each of these sets of boundary conditions correspond to a given stability of the flow, for a given filtering scheme (i.e. effective Reynolds number).

In passing from conditions A to B, it is easy to identify the cause of the increased instability: it is directly related to the increase of the transverse velocity at the boundary. This happens to be also reflected in a small, but visible, variation in the stream velocity difference at the lower boundary. More mysterious at first sight is the increased instability when comparing $\mathrm{B}$ and $\mathrm{C}$, for which there is no visible variation in the stream velocity difference at the boundary, and the transverse seed is the same. The key is to be found elsewhere than at the boundary. It lies in the pinching of the cold wake by the surrounding flow. This is seen by comparing the wakes for runs B2 and C1 (or C2) shown in Figs. 12 and 13 (or Fig. 14). One sees that changing the temperature modifies the collimation of the wake: the two vorticity layers are pushed one onto the other much more rapidly when the temperature is lower (run $\mathrm{C} 1$ ), which presumably increases the instability rate.

Finally, the increased stability when passing from $\mathrm{C}$ to $\mathrm{D}$ is easily explained in terms of accessible modes and seed amplitude: since the S-mode is not directly excited at the boundary by the symmetric $u_{\theta}$ profile, only the symmetric modes, which have a slower growth rate, grow first.

The collimation effect of the temperature was first observed in the simulations by Grappin et al. (1997); it was explained by invoking the different pressure heights of two hydrostatic columns in an atmosphere. This argument can be refined by considering the more general expression for the pressure derived from the Bernouilli law:

$\ln \left(P / P_{s}\right)=.5\left(\left(u_{s} / c\right)^{2}-(u / c)^{2}\right)+(G M / T)\left(1 / r-1 / r_{s}\right)(14)$

where the suffix $s$ denotes the point where base values are measured. We compare the pressure profiles for two steady radial flows with no interactions. If at a given distance, the pressure increases when the temperature is raised, then we conclude that the hot streams will pinch the cold streams. The expression $u / c$ is the Parker transonic solution, hence $(u / c)^{2}=g\left(r / R_{\star}\right)=f(r T)$, where $f$ is an universal function monotonically increasing $\left(R_{\star}\right.$ is the sonic point). Hence we see that the contributions of the kinetic and the potential energies are opposite: the kinetic energy leads to an overpressure of the slow (cold) streams, which is the standard Bernouilli effect, while the gravitational term leads to an overpressure of the hot streams, as argued in Grappin et al. (1997). Now, if one evaluates the respective ranges of both terms, it appears that the gravitational contribution is dominant in the accelerating region, which explains our results. Finally, if one considers varying the mean temperature, one indeed finds from Eq. (14) that the pinching grows when the temperature decreases.

Can we predict now the mixing length of a cold stream originating from a cold region in a non-magnetic corona? Let us consider the same parameters as in run B2. The divergence of the flow is (in our numerical units) $\operatorname{div} U^{0} \simeq 8$ : this measures the overall damping rate of the transverse fluctuations due to expansion. The advective or nonlinear rate, which in a standard Kelvin-Helmholtz problem gives the growth rate of the instability is (empirically) $k \delta U \simeq 30$. Hence the expansion should not matter, and the characteristic length of the instability should be $L_{\star} \simeq U^{0} /(k \delta U) \simeq 1 / 15$, which is clearly an order of magnitude below the observed mixing length in the case of run B2. It must be noted that the Mach number is close to one, which reduces the instability rate in the KelvinHelmholtz instability, but not in the required proportion. We must thus recognize that we are not able to predict quantitatively the instability rate or the mixing length. In particular, although we have identified the (apparent) origin of the increased instability when lowering the average temperature, namely the pinching of cold streams, we are not able to quantify the large change (by a factor ten) of the growth rate of the instability.

The problem of the origin of turbulence in the real solar wind is actually much complicated by the presence of the magnetic field. The main point is that, as soon as the Alfvén speed is larger than the velocity difference between streams, the transverse velocity fluctuations which 
are necessary to mix the streams are taken away in the form of (modified) Alfvén waves. Wake instabilities could be recovered in the vicinity of current sheets, as in the work by Einaudi et al. (1999). However, these authors considered only the homogeneous version of the problem, i.e., they did not take into account gravity, wind and stratification; the present work shows that the nature and parameters of the instability are different when taking into account the inhomogeneous wind. We are currently working on the question of the instability of magnetic and thermal wakes in the solar wind, using the same physical and numerical approach as in the present work.

Acknowledgements. We thank T. Zurbuchen and J.-L. Guermond for interesting and stimulating discussions, as well as I.D.R.I.S. for providing computer resources (project 990219).

\section{References}

Basdevant, C., Deville, M., Haldenwang, P., et al. 1986, Comput. Fluids, 14, 23

Coleman, P. J. Jr. 1968, ApJ, 153, 371

Einaudi, G., Boncinelli, P., Dahlburg, R. B., \& Karpen, J. T. 1999, J. Geophys. Res., 104, 521
Grappin, R., Velli, M., \& Mangeney, A. 1993, Phys. Rev. Lett., 70,2190

Grappin, R., \& Velli, M. 1966, J. Geophys. Res., 101, 425-444

Grappin, R. 1996, Onset of anisotropy and Alfven waves turbulence in the expanding solar wind, AIP Conf. Proc., 382, 306

Grappin, R., Léorat, J., Cavillier, E., \& Prigent, G. 1997, A\&A, 317, L31

Guermond, J.-L. 2000, Subgrid stabilization of Galerkin approximations of linear contraction semi-groups of class $C^{0}$ in Hilbert spaces, to appear in Int. J. Numer. Meth. P.D.E.

Habbal, S. R., Woo, R., Wineschi, S., et al. 1997, ApJ, 489, L103

Harten, A., Engquist, B., Osher S., \& Chekravanthy, S. R. 1997, J. Comput. Phys., 131, 3-47

Lele, S. K. 1992, J. Comput. Phys., 103, 16

Mangeney, A., Grappin, R., \& Velli, M. 1991, MHD turbulence in the solar wind, in Advances in Solar System Magnetohydrodynamics, ed. E. R. Priest, \& A. W. Hood (Cambridge University Press), 326-356

Passot, T., \& Pouquet, A. 1988, J. Comp. Phys., 75, 300

Poinsot, T. J., \& Lele, S. K. 1992, J. Comput. Phys., 101, 104

Roache, P. J. 1972, Computational Fluid Dynamics, Hermosa Publishers, Albuquerque, New Mexico

Siregar, E., Ghosh, S., \& Goldstein, M. L. 1995, Phys. Plasmas, 2,1480

Velli, M. 1994, ApJ, 432, L55 